\title{
Jewell-Sinclair theorem and the automatic continuity of a derivation
}

Amir A. Mohammed

Department of Mathematics

College of Education

University of Mosul

\section{Yassin Abdulljabar}

Depatrment of Mathematics

College of Com. And Math. Sci.

University of Mosul

\author{
Nadia A. Abdulraziq \\ Department of Mathematics \\ College of Education \\ University of Mosul
}

Received

25 / 01 / 2009
Accepted

05 / 05 / 2009

\section{مالخص}

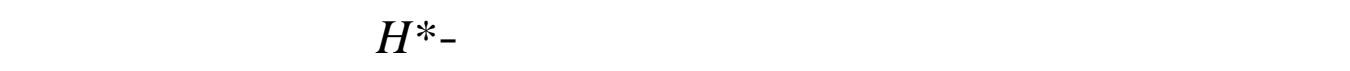

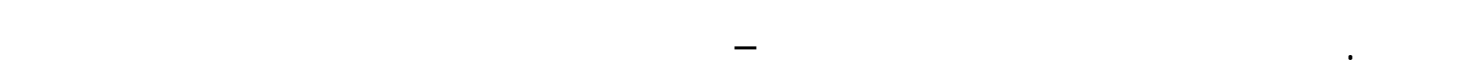
مبرهنة بيينا ممكن أيضاً ان تستنتج من صياغتنا لتك المبرهنة

\section{Abstract}

Villena's theorem ensure the automatic continuity of a derivation on a non-associative $H^{*}$-algebra with zero annihilator. In this paper, we formulate the Jewell-Sinclair theorem in a non-associative content, and we show that the Villena's theorem may be also followed from our formulation of such theorem.

\section{1- INTRODUCTION AND BASIC CONCEPTS:}

The range of derivations on Banach algebra are investigated, the fundamental work in this direction is due to Singer and Warmer in 1955 and it is in progress by another researchers, see [1],[2],[9] and [15].

Singer-Warmer theorem stated that: Let $A$ be a commutative Banach algebra, and let $\delta$ be a continuous derivation on $A$. Then $\delta(A)$ 
is contained in the radical of $A$. Singer and Warmer attained to improve the result mentioned above by delete the continuity of a derivation which is known as a Singer-Warmer conjecture: Let $A$ be a commutative Banach algebra and let $\delta$ be a derivation on $A$. Then $\delta$ is a continuous derivation on $A$ ?

Johnson in 1969 proved that every derivation on a semi-simple commutative Banach algebra with identity is continuous [6]. This result is a partial answer of Singer-Warmer conjecture because the algebra has an identity. Kaplansky in 1958 see [8] generalized Singer-Warmer conjecture as follows:

Is a derivation on semisimple Banach algebra be continuous? Johnson and Sinclair in 1968 see [7] confirmed Kaplansky conjecture, and the Johnson-Sinclair result it was generalized by Jewell and Sinclair in 1976 see [5] and Singer-Warmer conjecture confirmed by Thomas in 1988 see [13].

There are many studies of the automatic continuity of a derivation on non-associative Banach algebras. Particularly, the following problem is our interest. Is a derivation on a non-associative semisimple Banach algebra be continuous?

For details about this problems see [10], [12], [14], [15]. One of the important theorem in this direction also is due to Villena see [14] and it is our concern in this paper which is state that (Villena Theorem): Every derivation on $H^{*}$-algebra with zero annihilator is continuous.

Our work can be divided in three directions:

(1) State and study Jewell-Sinclair theorem: Every derivation on a Banach algebra $A$ is continuous if $A$ satisfies:

i- $A$ has no non-zero finite-dimensional nilpotent ideals.

ii- For each closed infinite-dimensional ideal $I$ of $A$, there is a sequence $\left\{a_{n}\right\}_{n \in N} \subseteq A$ such that the sequence $\left\{\left(a_{1} \cdot a_{2} \ldots \ldots a_{n}\right) I\right\}_{n \in N}$ of closed right ideals of $A$ is constantly decreasing.

(2) We put Jewell-Sinclair theorem on a non-associative content and we call it extended Jewel-Sinclair theorem as follows: Let $B$ be a semiprime Banach algebra not necessary associative such that for each closed infinite-dimensional ideal $I$ of $B$, there is a sequence $\left\{T_{n}\right\}_{n \in N} \subseteq M(B) \quad$ (the multiplication algebra of $B$ ) such that the sequence $\left\{\left(T_{1}, T_{2} \ldots \ldots T_{n}\right)^{-} I\right\}_{n \in N}$ of closed right ideals of $B$ is constantly decreasing. Then, any derivation on $B$ is continuous.

(3) We show that Villena's proof of the automatic continuity of a derivation on $H^{*}$-algebra with zero annihilator can be also followed by using extended Jewell-Sinclair theorem.

To explain our goal mentioned above we need the following definitions, propositions, and theorems. 
1-1 Definition [9], [11]: Let $A$ be an algebra. A derivation $\delta$ on $A$ is a linear mapping $\delta: A \rightarrow A$ such that:

$$
\delta(x y)=\delta(x) y+x \delta(y) \text { for all } x, y \in A \text {. }
$$

1-2 Definition [12]: Let $X$ and $Y$ be normed spaces and let $T$ be a linear mapping from $X$ into $Y$, then sparating subspace of $T$ (denoted by $Y_{T}$ ) defined as follows: $Y_{T}=\left\{y \in Y: \exists\left\{x_{n}\right\} \subseteq X,\left\{x_{n}\right\} \rightarrow 0, T\left\{x_{n}\right\} \rightarrow y, \forall n \in N\right\}$.

1-3 Proposition [3], [12]: Let $A$ be a normed algebra and $\delta$ be a derivation on $A$. Then $A_{\delta}$ is closed ideal of $A$.

1-4 Sinclair Theorem [12]: Let $X$ and $Y$ be Banach spaces and let $T: X \longrightarrow Y$ be a linear mapping. If the sequences $\left\{S_{n}\right\}_{n \in N} \subseteq B L(X)$ and $\left\{R_{n}\right\} \subseteq B L(Y)$ satisfy $T S_{n}=R_{n} T$, then there is an integer $k \in N$ such that $\left(R_{1} \ldots . . R_{n} Y_{T} \overline{)}=\left(R_{1} \ldots \ldots R_{k} Y_{T} \overline{)} \quad \forall n \geq k\right.\right.$.

We recall from [4] that, an algebra $A$ is semiprime if any ideal $I$ of $A$ such that $I^{2}=0$ implies $\mathrm{I}=0$. Also an annihilator of $A$ (denoted by Ann(A)) defined as follows: $A n n(A)=\{x \in A: a x=x a=0, \forall a \in A\}$, and we say that $A$ is of zero annihilator if $A n n(A)=\{0\}$. Finally, the multiplication algebra of $A$ denoted by $M(A)$ is defined as a subalgebra of $L(A)$ (the algebra of all linear mapping on $A$ ) generated by following operators:
$I d_{A}: A \rightarrow A$ $L_{x}: A \rightarrow A$
, $R_{x}: A \rightarrow A$
$a \mapsto I d_{A}(a)=a$
$a \mapsto L_{x}(a)=x a$
$a \mapsto R_{X}(a)=a x$

called identity, left, and right multiplication operators respectively.

\section{2- EXTENDED JEWELL-SINCLAIR THEOREM .}

We extending the Jewell-Sinclair theorem in the following way.

2-1 Extended Jewell-Sinclair Theorem: Let $B$ be a semiprime Banach algebra not necessary associative such that for each closed infinitedimensional ideals $I \subseteq B$, there is a sequence $\left\{T_{n}\right\}_{n \in N} \subseteq M(B)$ such that the sequence $\left\{\left(T_{1} \cdot T_{2} \ldots \ldots T_{n}\right) I\right\}_{n \in N}$ of closed right ideals of $B$ is constantly decreasing. Then, any derivation on $B$ is continuous.

\section{Proof :}

Let $B$ be a semiprime Banach algebra not necessary associative and let $\delta$ be a derivation on $B$. By proposition (1-3), $B_{\delta}$ is closed ideal of $B$. If $B_{\delta}$ is infinite-dimensional, then by assumption there is a sequence $\left\{T_{n}\right\}_{n \in N} \subseteq M(B)$ such that the sequence $\left\{\left(T_{1} \cdot T_{2} \ldots \ldots T_{n}\right) B_{\delta}\right\}_{n \in N}$ is constantly decreasing. By applying Sinclair theorem (1-4) and by setting:

$X=Y=B, \quad T=\delta, \quad R_{n}=T_{n}=S_{n}$

We get, there exist a natural number $K \in N$ such that:

$\left(T_{1} \ldots \ldots T_{n} B_{\delta} \overline{)}=\left(T_{1} \ldots . . T_{K} B_{\delta}\right) \forall n \geq K\right.$. This condition implies that $\left\{T_{n}\right\}_{\text {noN }}$ not constantly decreasing, and this is a contradiction. So, $B_{\delta}$ must be finite-dimensional. Note that, if $\mathrm{B}_{\delta}$ is finite-dimensional, then $\left.\delta\right|_{B_{\delta}}$ is continuous. Now we claim that $B_{\delta}^{2}=\{0\}$. Let $a, b \in B_{\delta}$, 
then there is $\left\{a_{n}\right\}_{n \in N} \subseteq B$, such that $\operatorname{Lim}_{n \rightarrow \infty} a_{n}=0$ and $\operatorname{Lim}_{n \rightarrow \infty} \delta\left(a_{n}\right)=a$. Now, $\operatorname{Lim}_{n \rightarrow \infty} \delta\left(a_{n} b\right)=\operatorname{Lim}_{n \rightarrow \infty} \delta\left(a_{n}\right) b+\operatorname{Lim}_{n \rightarrow \infty} a_{n} \delta(b)$. Since $\operatorname{Lim}_{n \rightarrow \infty} a_{n}=0$ and $b \in B_{\delta}$, and that $B_{\delta}$ is an ideal of $B$, we have $\operatorname{Lim}_{n \rightarrow \infty} a_{n} b=0, a_{n} b \in B_{\delta}$ and take into account that $B_{\delta}$ finite-dimensional and $\left.\delta\right|_{B_{\delta}}$ is continuous we get $\operatorname{Lim}_{n \rightarrow \infty} \delta\left(a_{n} b\right)=0$. Now, $a b=\operatorname{Lim}_{n \rightarrow \infty} \delta\left(a_{n}\right) b=\operatorname{Lim}_{n \rightarrow \infty}\left[\delta\left(a_{n} b\right)-a_{n} \delta(b)\right]=0$, we find that $B_{\delta}^{2}=\{0\}$ and since $B$ is semiprime by assumption, so we have $B_{\delta}=\{0\}$ and by using closed graph theorem we obtain that $\delta$ is continuous. This completes the proof

\section{3- AN APPLICATION EXAMPLE .} theorems.

Before we give our example we need some propositions and Recall from [4] that an involution of an algebra $A$ is a mapping $x \rightarrow x^{*}$ of $A$ into $A$ such that the mapping * satisfies the following conditions:

For all $x, y \in X, \alpha \in C$ (complex field)

(i) $(x+y)^{*}=x^{*}+y^{*}$

(ii) $(\alpha x)^{*}=\alpha^{*} x^{*}$

(iii) $\left(x^{*}\right)^{*}=x$

(iv) $(x y)^{*}=y^{*} x^{*}$

Also, recall that from [11] if $A$ is a semiprime algebra and if $C(A)$ is the extended centroid of $A$, then $A$ is said to be centrally closed if and only if $C(A)$ is equal to the base field.

3-1 Proposition [14]: Let $\delta$ be a derivation on a topologically simple Banach algebra $A$ not necessary associative, and suppose that there exists non zero $T$ in $M(A)$ with finite dimensional range. Then $\delta$ is continuous.

3-2 Proposition [14]: Let $A$ be a centrally closed prime algebra such that $\operatorname{dim}(T(A))>1$ for all non zero $T$ in the multiplication algebra $M(A)$ of $A$. Then there is a sequence $\left\{b_{n}\right\}_{n \in N}$ in $A$ and $\left\{T_{n}\right\}_{n \in N}$ in $M(A)$ such that $T_{n} \ldots T_{1} b_{n} \neq 0$ and $T_{n+1} \ldots T_{1} b_{n}=0$ for all $n$ in $N$.

3-3 Theorem [14]: Every $H^{*}$-algebra with zero annihilation is the closure of the orthogonal sum of its minimal closed ideal, and these are topologically simple $H^{*}$-algebra.

3-4 Theorem [14]: Every topologically simple $H^{*}$-algebra is centrally closed prime algebra.

3-5 Lemma [11]: Let $A$ be an algebra, and assume the existence of a non-degenerate symmetric associative bilinear form $<\ldots$, . $>$ on $A$. Then we have: 
(i) There exist a unique linear algebra involution \# on the multiplication algebra $M(A)$ of $A$ satisfying $L_{d}^{\#}=R_{d}$ and $R_{d}^{\#}=L_{d}$ for all $d \in A$

(ii) for $\mathrm{x}, \mathrm{y}$ in $A$ and $T$ in $M(A)$. The equality $\left.\langle T x, y\rangle=<x, T^{\#}(y)\right\rangle$ holds.

Now, we present our application example.

3-6 Example (Villena's Theorem): Every derivation on $H^{*}$-algebra $A$ with zero annihilator is continuous.

\section{Proof:}

Let $\delta$ be a derivation on $H^{*}$-algebra $A$ with zero annihilator. We assume first that $A$ is topologically simple, and by applying theorem (3-4) we have, $A$ is a centrally closed prime algebra. Now, $M(A)$ satisfying one of the following case:

(i) There exist an element in $M(A)$ has finite dimensional range.

(ii) Every element in $\mathrm{M}(\mathrm{A})$ has infinite dimensional range.

First case: if (i) is true, then $\delta$ is continuous by using proposition (3-1).

Second case: If every element in $M(A)$ has infinite-dimensional range, then by using proposition (3-2) there exist a sequence $\left\{C_{n}\right\}_{n \in N} \subseteq A$ and $\left\{T_{n}\right\}_{n \in N} \subseteq M(A)$ such that:

$T_{n+1} T_{n} \ldots T_{1} C_{n}=0$

$T_{n} T_{n-1} \ldots T_{1} C_{n} \neq 0$

For all $n$ in $N$. By using the fact that every topologically simple $H^{*}$ algebra contain a non degenerate symmetric associative bilinear continuous form $\langle.,$.$\rangle , also by lemma (3-5), there exist algebra$ involution \# on $M(A)$ such that :

$$
L_{b}^{\#}=R_{b} \quad, \quad R_{b}^{\#}=L_{b}, \forall b \in A
$$

Now, suppose that $\mathrm{n} \in \mathrm{N}$ is a positive integer number satisfying :

$\overline{T_{1}^{\#} \ldots T_{n}^{\#}(A)}=\overline{T_{1}^{\#} \ldots T_{n+1}^{\#}(A)}$

This implies that a sequence $\left\{T_{n}^{\#}\right\}_{n \in N} \subseteq M(A)$ is not constantly decreasing .

Now, from equation (1) we can have $T_{n+1} T_{n} \ldots T_{1} C_{n}=0$.

The mapping $<$. . . $>$ is non degenerate, continuous [by lemma (3-5)] and from equation (4) we can get:

$$
\begin{aligned}
0 & =<A, T_{n+1} \ldots T_{1}\left(C_{n}\right)> \\
& =<A,\left(\left(T_{n+1} \ldots T_{1}\right)^{\#}\right)^{\#}\left(C_{n}\right)> \\
& =<\left(T_{n+1} \ldots T_{1}\right)^{\#}(A),\left(C_{n}\right)> \\
& =<T_{1}^{\#} \ldots T_{n+1}^{\#}(A), C_{n}> \\
& =<T_{1}^{\#} \ldots T_{n+1}^{\#}(A), C_{n}> \\
& =<T_{1}^{\#} \ldots T_{n}^{\#}(A), C_{n}> \\
& =<T_{1}^{\#} \ldots T_{n}^{\#}(A), C_{n}> \\
& =<A,\left(T_{1}^{\#} \ldots T_{n}^{\#}\right)^{\#} C_{n}> \\
& =<A, T_{n} \ldots T_{1} C_{n}>
\end{aligned}
$$


This is a contradiction because $T_{n} T_{n-1} \ldots T_{1} C_{n} \neq 0$. Now, for every $\mathrm{n} \in N$ there exist sequence $\left\{\overline{T_{1}^{\#} \ldots T_{n}^{\#}(A)}\right\}_{n \in N}$ of closed right ideal of $A$ constantly decreasing. Since $A$ is semiprime, applying extended Jewell-Sinclair theorem we get $\delta$ is continuous. In order to obtain general case of the proof assume that $A$ has zero annihilator. Now, let $M$ be a minimal closed ideal of $A$, then $M$ is a part of direct sum of $A$. If the inclusion $\delta(M) \subseteq M$ is not true then there exist a non zero minimal closed $J$ of $A$ such that $\delta(M) \phi J$, and $J \cap M=\{0\}$. This is a contradiction because $A$ is semiprime, then the inclusion must be $\delta(M) \subseteq M$, or $\delta(M) \subseteq J \cap M \subseteq M$. And since $M$ is topologically simple by theorem (3-3), it follows from the first part of the proof that $\delta$ is continuous on $M$. Let $x 0 A_{\delta}$, then the exist $\left\{x_{n}\right\} \subseteq A$ such that $\operatorname{Lim}_{n \rightarrow \infty} x_{n}=0$ and $\operatorname{Lim}_{n \rightarrow \infty} \delta\left(x_{n}\right)=x$. Now, for all $y \in M$ and $\operatorname{Lim}_{n \rightarrow \infty} x_{n}=0, y x_{n} \in M$ implies $\operatorname{Lim}_{n \rightarrow \infty} \delta\left(y x_{n}\right)=0$ become $\delta$ is continuous on $M$. Now $\operatorname{Lim}_{n \rightarrow \infty} \delta\left(y x_{n}\right)=\operatorname{Lim}_{n \rightarrow \infty}\left[\delta(y) x_{n}+y \delta\left(x_{n}\right)\right]$. This implies $0=y x$. Similarity we can get $0=x y$. For all $x \in A_{\delta}$, and $y \in M$, we have $0=A_{\delta} M=M A_{\delta}$ for each minimal closed ideal $M$ of $A$, and hence 0 $=A A_{\delta}=A_{\delta} A$ which implies that $A_{\delta} \subseteq A n n(A)=\{0\}$. Using closed graph theorem we get $\delta$ is continuous

\section{References}

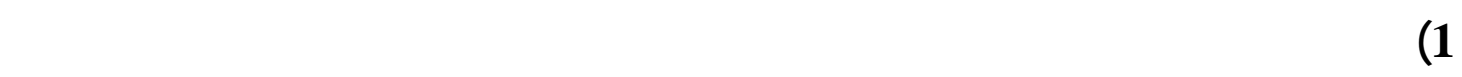

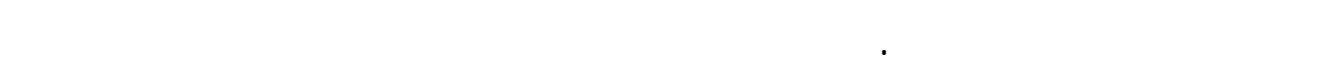
العراق، المجلد 32، 3(2008)، 153 -159.

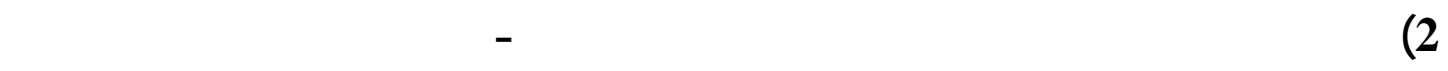

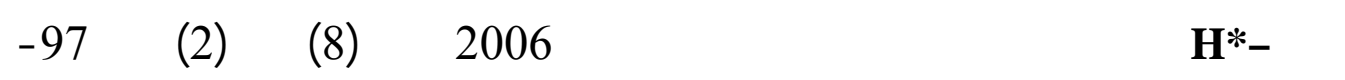
.98

3) Assadallah, N. "Closable derivations of simple C*-Algebras", Glasgow Math. J. 24 (1988), 181-183.

4) Bonsall, F. F., Duncan, J. "Complete Normed Algebras", Springerverlag, Berlin. Heidelberg New York, (1973).

5) Jewell, N. P., Sinclair, A. M. "Epimorphosis and derivations on $L_{1}(0,1)$ are continuous", Bull Lnodon Math, Soc. 8 (1976), 135139.

6) Johnson, B. E. "Continuity of derivations on commutative Banach algebras". Amer. J. Math., 91 (1969), 1-10. 
7) Johnson, B. E., Sinclair, A. M. "Continuity of derivations and a problem of Kaplansky". Amer. J. Math., 90 (1968), 1067-1073.

8) Kaplansky, I. "Derivations. Banach algebras seminar on analytic functions". Vol. II Institute for Advanced Study, Princeton, 1958.

9) Palmar, T. W. "Banach algebras and the general theory of *algebra", Combridge Uni. Press, (1994).

10) Rodriguez, A. "Jordan structures in analysis. In: Jordan, Algebras", Proc. Conf. Oberwlfach, August 9-15 (1992), W. Kaup. K. M. C. Crimman and Peterson (eds.), de Grugter, Berlin (1994), 97-186.

11) Rodriguez, A. P. "Continuity of densely valued homeomorphisms into H*-algebras", Quart. J. Math. Oxford (2)-46 (1995), 107-118.

12) Sinclair, T. M. "Automatic Continuity of Linear Operators", Cambridge Uni. Press, (1976).

13) Thomas, M. P. "The image of derivations is contained in the radical", Ann of Math., 128 (1988), 435-460.

14) Villena, A. R. "Continuity of derivations on $H^{*}$-algebras", Proc. Amer. Soc. Vol. 122, 3, (1994), 821-826.

15) Villena, A. R. "Automatic Continuity in associative and nonassociative context", Irish Math. Soc. Bulletin 46 (2001), 43-76. 